\title{
Effect of Low Level Laser Therapy on Postoperative Pain After Single Visit Endodontic Treatment: A Placebo-Controlled Randomized Clinical Trial
}

\author{
Elif Bahar Çakıcı ${ }^{1}$, Adem Günaydın ${ }^{1}$, Büşra Uysal ${ }^{1}$, Fatih Çakıcı1 \\ ${ }^{1}$ Department of Endodontics, Faculty of Dentistry, Ordu University, Ordu, Turkey \\ Received: 14 March 2020, Accepted: 06 April 2020, Published online: 31 August 2020 \\ (C) Ordu University Institute of Health Sciences, Turkey, 2020
}

\begin{abstract}
Objective: To evaluate the effect of low-level laser therapy (LLLT) on postoperative pain after root canal treatment.

Methods: This study was designed as a randomized, single-blinded, placebo-controlled trial of 2 groups. Forty-two patients were included in the study according to the inclusion and exclusion criteria. The treatment procedures were performed by one operator. After local anesthesia and rubber dam isolation, access cavity preparation was performed by diamond burs with high-speed handpieces under the water cooling. Instrumentation of procedures was performed by Reciproc R50 files. Irrigation protocol was completed, canals were dried, filled with gutta-percha cones and AH Plus sealer. After chemo- mechanical instrumentation and root canal filling procedures, LLLT were applied for 60 seconds per tooth using $\mathrm{Nd}$ YAG laser ( $\lambda=1064 \mathrm{~nm}, 100 \mathrm{~mJ}, 10 \mathrm{~Hz}, 1-\mathrm{W}$ ) The same procedures as in the laser group were performed, been completed but the laser was not activated in this group. These patients were assigned as a placebo group. Postoperative pain was measured and documented via the Visual Analogue Scale.

Results: The independent sample t-test was performed after normality and homogeneity tests. No statistically significant differences were detected between the groups in terms of demographic data (age and tooth number) $(\mathrm{P}>$.05). No statistically significant difference was found between the groups at the 4th, 8th, 48th, and 72nd hours ( $\mathrm{p}>.05)$. However, there was a statistically significant difference between groups at the 12th $(\mathrm{p}=.030)$ and 24th hours $(\mathrm{p}=.041)$.
\end{abstract}

Conclusion: LLLT can decrease postoperative pain after root canal treatment of single-rooted teeth.

Key words: Biostimulation, irreversible pulpitis, low level laser, single visit

Suggested Citation: Cakici EB, Gunaydin A, Uysal B, Cakici F. Effect of Low-Level Laser Therapy on Postoperative Pain After Single Visit Endodontic Treatment: A Placebo-Controlled Randomized Clinical Trial. Middle Black Sea Journal of Health Science, 2020; 6(2):166-171.

Address for correspondence/reprints:

Adem Günaydın

E-mail: gunaydnadem@gmail.com

Telephone number: +90 (541) 3627806

DOI:

ORCID-ID 0000-0002-3300-3342 


\section{Introduction}

Irreversible pulpitis is one of the most common reasons requiring root canal treatment (Boykin et al., 2003). However, root canal treatment may result in post-operative pain (Asnaashari et al., 2011). Various factors including the number of visits, the use of intracanal medicament, pulpal and periapical situation affect the formation of a flare-up (DiRenzo et al., 2002). Moreover, host factors such as age, gender, presence of periapical-induced preoperative pain, dental group, primary treatment or retreatment, and irritants inside the root canal system contribute to the increase in the frequency of flare-up. Furthermore, iatrogenic factors, for instance, debris extrusion in the periapical area, and deterioration of apical patency during preparation increase the incidence of flare-ups (Mashiko et al., 1985)

Attar et al., (2008) have recommended nonsteroidal anti-inflammatory drugs, corticosteroids, and antibiotics to deal with undesired postoperative complications. Low-level laser therapy (LLLT) has been introduced in endodontics as a non-invasive non-pharmacotherapeutic and simple manipulation technique (Asnaashari et al., 2013). The main effects of this laser therapy include anti-inflammatory features, immune stimulation, analgesia, and induction of cell proliferation. LLLT increases phagocytosis synthesis, lymphatic drainage, and vasodilatation while reducing bradykinin synthesis and histamine release which mechanisms affect the inflammatory process (Rochkind et al., 1989). Additionally, LLLT provides faster tissue healing by rising of vascularization, support growth of fibroblasts and collagen formation, and changes in immunological reaction. According to the results of a systematic review and meta-analysis, LLLT also retards the beginning of pain, decreases both intensity and duration of pain after endodontic treatment and the use of analgesics is relatively reduced, even in some cases analgesic drugs are not needed (He et al., 2013). This study aimed to evaluate the effect of LLLT on postoperative pain after root canal treatment. The null hypothesis was that there is no difference between the groups in postoperative pain.

\section{Methods}

\section{Study Design}

This study was designed as a randomized, singleblinded, placebo-controlled trial of 2 groups.

\section{Patients and Inclusion/Exclusion Criteria}

The study was approved by the local ethics committee of Ordu University, and informed consent was received from all participants. Study subjects were selected among patients who presented to the Department of Endodontics, Faculty of Dentistry of Ordu University from September 2017 to March 2018.

Root canal treatment was planned for patients who diagnosed asymptomatic irreversible pulpitis and have a single root and single canal. Pulp vitality was assessed with cold spray (Endo ice refrigerant spray, Coltene/Whaledent Inc., Mahwah, NJ). The patient who included this study reported no preoperative pain and all selected teeth were vital. Adequate root canal fillings were included, and the extruded fillings were not considered in the study. Patients aged 15 to 45 years. Exclusion criteria related to teeth were swelling or sinus tract, acute pain, periodontal probing greater than $3 \mathrm{~mm}$, internal and external resorption, fractured and cracked tooth, percussion sensitivity, and periapical index classification 3, 4, 5 according to Orstavik et al. (1986). Exclusion criteria related to the systemic health of patients included diabetes, hypertension, and cardiovascular pathologies, the use of analgesics and/or antibiotics at least one week before treatment and using antidepressant drugs.

\section{Sample Size Calculation}

A pilot study was conducted to calculate the sample size of the study. Therefore, twenty patients were randomly assigned to two groups. The same protocol was used both in the pilot study and the main study. According to the results of the pilot study, a sufficient number of the sample was found as 38 patients for two 2 groups (power $=80 \%$, significance level $=0.05$, effect size $=0.80$ ). Assuming that approximately $10 \%$ of patients may not respond, 21 patients were assigned to each group to ensure a representative sample.

\section{Treatment Procedures}

The treatment procedures were performed by one operator (G.A.). Initially, patients' age, gender, and tooth number were recorded by the operator. $1.5 \mathrm{~mL}$ $2 \%$ articaine with $0.012 \mathrm{mg}$ epinephrine (Ultracaine DS Forte; Aventis, Istanbul, Turkey) was used as a local anesthetic. After rubber dam isolation, access cavity preparation was performed by diamond burs (ADIA Dental Burs, Istanbul, Turkey) with highspeed handpieces under the water cooling. A \#10 Ktype (Kerr Corporation, Orange, CA) file was inserted to root canal to determine working length using an apex locator (Raypex, VDW, Munich, Germany). The working length was set as $1 \mathrm{~mm}$ shorter than the apical foramen and confirmed with periapical 
radiographs. Instrumentation of procedures was performed by Reciproc R50 files (VDW, Munich, Germany). Irrigation protocol was completed $5 \mathrm{~mL}$ 17\% EDTA (Werax, Izmir, Turkey) and $15 \mathrm{ml} 2.5 \%$ sodium hypochlorite $(\mathrm{NaOCl})$ (Wizard, Istanbul, Turkey) with a side-vented needle (NaviTip needle; Ultradent Products Inc, South Jordan, UT). Subsequently, canals were dried with paper points and filled with gutta-percha cones and AH Plus sealer (Dentsply Maillefer, Tulsa, OK). Lateral cold condensation technique was used for obturation. Finally, the coronal access cavity was restored with temporary restorative material (Cavit G; 3M ESPE, St Paul, MN). It may be the effect of dental matrix bands on postoperative pain so permanent restoration was completed after the end of the experiment.

\section{Randomization}

Randomization was produced using a website (http://www.random.org) after the clinician (G.A.) was completed all treatment procedures. All procedures were conducted by one clinician (C.E.B.), and the assignment was concealed from the clinician who performed the laser applications (U.B.). Data analysis and interpretation were completed by the other researcher (C.F.).

\section{Laser Group}

After chemo- mechanical instrumentation and root canal filling procedures, LLLT was applied for 60 seconds per tooth using Nd-YAG laser $(\lambda=1064$ $\mathrm{nm}, 100 \mathrm{~mJ}, 10 \mathrm{~Hz}, 1-\mathrm{W}$ [Deka smart file, DEKA, Italy]). The application of the laser was performed through the root canal and to the buccal mucosa over the apices of the target tooth. An application biostimulation tip was used to ensure a constant distance of $10 \mathrm{~mm}$ to the tissue.

\section{Placebo Group}

The same procedures as in the laser group were performed, but the laser was not activated in this group. These patients were assigned as a placebo group.

\section{Pain Evaluation}

Postoperative pain was measured and documented via a visual analog scale (VAS). VAS consists of a $100 \mathrm{~mm}$ line which is represented at one end by a sign 'no pain' and at the other end 'unbearable pain' (Figure 1). This form was given to each patient and they were instructed to mark according to the pain intensity at 4th, 8th, 12th, 24th, 48th and 72nd hours. Name:

Surname

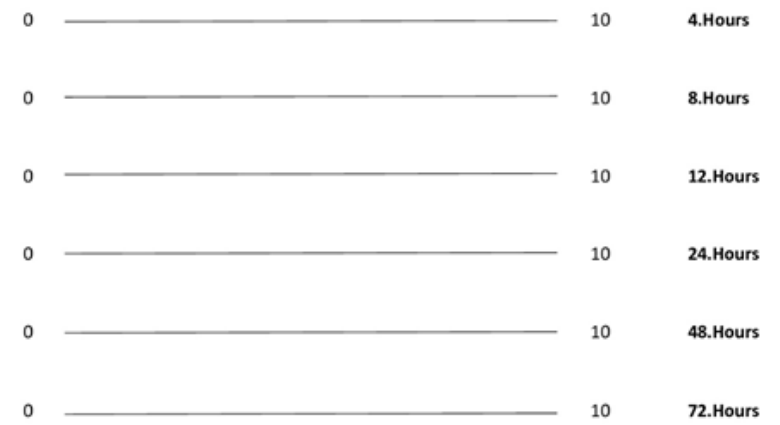

Please indicate your pain score on erery time intervals

Figure 1. Visual Analog Scale

\section{Statistical Analysis}

The data were expressed displayed as mean \pm standard deviation. The independent sample t-test was performed after the normality and homogeneity tests. A Chi-square test was performed in the analysis of the nominal data. $\mathrm{P}<0.05$ values were considered statistically significant. Statistical tests were performed with SPSS (Version 24.0, SPSS Inc., Chicago, IL).

\section{Results}

Table 1 shows descriptive statistics and postoperative sensitivity at different evaluation points. Figure 2 indicates the participation of patient numbers for each group. Aside from sex, no statistically significant differences were detected between the groups in terms of demographic data (age and tooth number) $(\mathrm{P}>.05)$. There was a statistically significant difference between groups at the 12th and 24 th hours $(\mathrm{p}<.05)$. LLLT resulted in lower pain levels than the placebo group at 12nd and 24th hours. However, no statistically significant difference was found between the groups at the 4th, 8th, 48th, and 72nd hours ( $p>.05$ ). During this study, no patient reported swelling, sinus tract, palpation pain and didn't need analgesics both in groups. 


\section{Effect of Low Level Laser Therapy on Postoperative Pain}

Table 1. Descriptive statistics and the results of statistical analysis regarding postoperative sensitivity in the laser and placebo groups at different evaluation points

\begin{tabular}{lccc}
\hline Study Details and Pain Levels & LLLT Group $(\mathbf{n = 1 5})$ & Placebo Group $(\mathbf{n = 1 9})$ & P Value \\
\hline Demographic data & & & $>, 05$ \\
Age $\quad$ Men (n) $\quad$ Women (n) & $30,86 \pm 11,82$ & $25,52 \pm 10,10$ & $>, 05$ \\
Sex & 12 & 11 & $>, 05$ \\
Postoperative pain levels at 4th hours & $0,753 \pm 1,165$ & $1,526 \pm 2,166$ & $>, 05$ \\
Postoperative pain levels at 8th hours & $1,013 \pm 1,744$ & $1,489 \pm 1,905$ & $<, 05^{*}$ \\
Postoperative pain levels at 12th hours & $0,266 \pm 0,158$ & $1,231 \pm 1,736$ & $<, 05^{*}$ \\
Postoperative pain levels at 24th hours & $0,273 \pm 0,127$ & $0,847 \pm 1,144$ & $>, 05$ \\
Postoperative pain levels at 48th hours & $0,266 \pm 0,129$ & $0,731 \pm 1,170$ & $>, 05$ \\
Postoperative pain levels at 72nd hours & $0,306 \pm 0,212$ & $0,678 \pm 1,163$ & \\
\hline
\end{tabular}

* statistical difference $(\mathrm{P}=0,05)$

\section{CONSORT}

TRANSPARENT REPORTING of TRIALS

CONSORT 2010 Flow Diagram

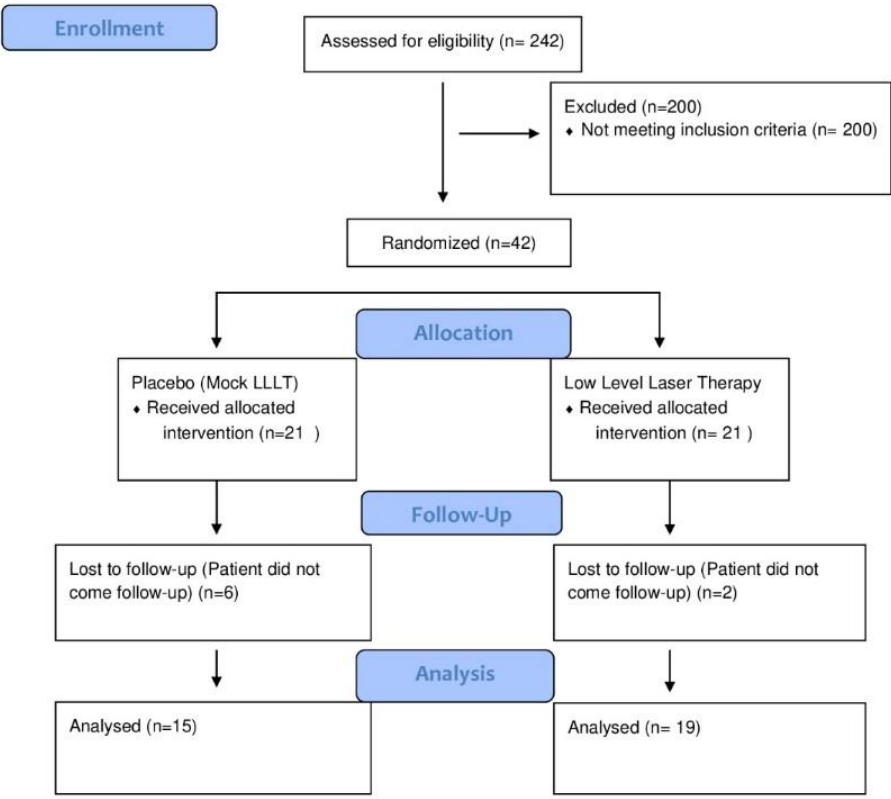

Figure 2. CONSORT diagram summarizing patients eligible for the study 


\section{Discussion}

Postoperative pain after root canal treatment is an unpleasant situation both for patients and clinicians. The most common postoperative pain control agents include antibiotics, non-steroidal anti-inflammatory agents, and corticosteroids which can lead to undesirable effects in some patients who are pregnant or have a drug allergy (Attar et al., 2008a). Recently, LLLT has been considered as a helpful method to reduce post-op pain associated with root canal treatment due to its effective and nonpharmacological character. Researchers have described the main effects of LLLT as an antiinflammatory, immune-stimulating, analgesic, and cell proliferation-inducing effects (Kreisler et al., 2004). When photons' light interacts with the cellular structure, its energy is absorbed by the cells, producing the expected therapeutic effect on tissues (Asnaashari et al. 2013a). The increased cellular energy of periapical tissue results in pain relief, nerve regeneration, wound healing, and immune system modulation (Barden et al., 2004).

In a study conducted by Asnaashari et al. (2011a), significant pain reduction was seen at 4, 8, 12 and 48 hours after the endodontic treatment in the LLLT group. Pawar et. al. (2014) reported the pain reduction effect of LLLT after the endodontic treatment of single root premolars. They concluded that reduction of pain was statistically significant in the LLLT group at 4th and 8th hours, but no statistically significant difference was found in pain reduction at 24th and 72nd hours. Arslan et al. (2017) examined the effect of LLLT on postoperative pain after root canal retreatment. Although they reported less pain in the LLLT group than in the placebo group in the first 4 days, they have found no statistically significant differences after 5 and 7 days. The null hypothesis was that there is no difference between the groups in postoperative pain. According to the results of the present study, significantly lower pain was found in the LLLT group at 12th and 24th hours between the groups, but no significant difference was found 8th, 48th, and 72nd hours. Thus, the null hypothesis was rejected. These differences between the results may originate from various factors such as using the type of laser, power of laser, inclusion criteria of the patient in studies.

The decrease in pain between the groups was similar after 24 hours, and no significant difference was found between 48 and 72 hours. According to the manufacturer, the complete disappearance of the effect of articaine varies between 60-225 min, so there was no significant difference between the groups in the first 8 hours. Alonso et al. (2012) reported that most of the pain occurred at 6th and 18th hours in the single visit root canal treatment which was significantly decreased after 24th hours. This result agrees with the findings of our study which no significant difference was found after 24th hours between LLLT and placebo groups.

According to the systematic review by Manfredi et al. (2016), there is no evidence to suggest that single-visit or multiple-visit root canal treatment is better than the other. On the other hand, there is a tendency for clinicians to perform single-visit endodontic treatment recently because of the benefits such as not requiring additional anesthetic injections, no need replacement of the rubber dam or intracanal medication, absence of inter visit leakage, loss of temporary seal, or any of the accidents that can and do occur between the visits (Attar et al., 2008b). Therefore, all root canal treatments were completed in single-visit in the present study.

\section{Conclusion}

Within the limitations of the present study, LLLT can decrease postoperative pain after root canal treatment of single-rooted teeth.

Ethics Committee Approval: The study was approved by the ethics committee of Ordu University (Decision No: 2017/136). The study was performed following the aid of the ethical standards down in the 1964 Declaration of Helsinki and its later amendments.

Peer-review: Externally peer-reviewed.

Author Contributions: Concept- C.E.B.; DesignG.A., C.F.; Materials- C.E.B., U.B.; Data Collection and Processing- G.A., U.B.; Literature ReviewC.E.B., C.F.; Writing- G.A., U.B.; Critical ReviewC.E.B., G.A.

Conflict of Interest: No conflict of interest was declared by the authors.

Financial Disclosure: The authors declared that this study hasn't received no financial support. 


\section{References}

Alonso-Ezpeleta LO, Gasco-Garcia C, CastellanosCosano L, Martín-González J, López-Frías FJ, Segura-Egea JJ. Postoperative pain after one-visit root-canal treatment on teeth with vital pulps: Comparison of three different obturation techniques. Med Oral Patol Oral Cir Bucal. 2012;17(4).

Arslan H, Doğanay E, Karataş E, Ünlü MA, Ahmed HMA. Effect of Low-level Laser Therapy on Postoperative Pain after Root Canal Retreatment: A Preliminary Placebo-controlled, Triple-blind, Randomized Clinical Trial. J Endod. 2017;43(11):1765-9.

Asnaashari M, Mohebi S, Paymanpour P. Pain Reduction Using Low-Level Laser Irradiation in Single-Visit Endodontic Treatment. J Lasers Med Sci. 2011;2(4):139-43.

Asnaashari M, Safavi N. Application of Low-level Lasers in Dentistry (Endodontic). Rev Artic J Lasers Med Sci. 2013;4(2):57-66.

Attar S, Bowles WR, Baisden MK, Hodges JS, McClanahan SB. Evaluation of Pretreatment Analgesia and Endodontic Treatment for Postoperative Endodontic Pain. J Endod. 2008;34(6):652-5.

Barden J, Edwards JE, McQuay HJ, Moore RA. Pain and analgesic response after third molar extraction and other postsurgical pain. Pain. 2004;107(12):86-90.

Boykin MJ, Gilbert GH, Tilashalski KR, Shelton BJ. Incidence of endodontic treatment: A 48-month prospective study. J Endod. 2003;29(12):806-9.

DiRenzo A, Gresla T, Johnson BR, Rogers M, Tucker D, BeGole EA. Postoperative pain after 1- and 2visit root canal therapy. Oral Surg Oral Med Oral Pathol Oral Radiol Endod. 2002;93(5):605-10.

He WL, Li CJ, Liu ZP, Sun JF, Hu ZA, Yin X, et al. Efficacy of low-level laser therapy in the management of orthodontic pain: A systematic review and meta-analysis. Lasers Med Sci. 2013;28(6):1581-9.

Kreisler MB, Al-Haj H, Noroozi N, Willershausen B, Dhondt B. Efficacy of low-level laser therapy in reducing postoperative pain after endodontic surgery - A randomized double-blind clinical study. Int J Oral Maxillofac Surg. 2004;33(1):3841.

Manfredi M, Figini L, Gagliani M, Lodi G. Single versus multiple visits for endodontic treatment of permanent teeth (Review) Summary of findings for the main comparison. 2016;(12).
Mashiko K, Kanuma A, Kozawa T, KiWon Lee, Wu A, Zhihua Wang. Academia-industry collaboration in SoC design education wishes and reality. Proc 2004 IEEE Asia-Pacific Conf Adv Syst Integr Circuits [Internet]. 1985;11(11):1821.

Orstavik D, Kerekes K, Eriksen HM. The periapical index: A scoring system for radiographic assessment of apical periodontitis. Dent Traumatol. 1986;2(1):20-34.

Pawar SS, Pujar MA, Makandar SD, Kaiser MI. Post endodontic treatment pain management with lowlevel laser therapy. 2014;8(2):60-3.

Rochkind S, Rousso M, Nissan M, Villarreal M, BarrNea L, Rees DG. Systemic effects of low-power laser irradiation on the peripheral and central nervous system, cutaneous wounds, and burns. Lasers Surg Med. 1989;9(2):174-82. 